\title{
Multi-Attribute Double Auction Models for Resource Allocation in Computational Grids
}

\author{
Wanglin Kang*, Lei Wang and Wencheng Yu \\ College of Economics and Management, Shandong University of Science and Technology, China
}

\begin{abstract}
In this paper we propose the double auction allocation models for computational grids, and two double auction algorithms for resource allocation: Single Price Double Auction algorithm (SPDA), and Multiple Attribute Double Auction algorithm (MADA). The fairness and comprehensive performance are introduced into the traditional double auction models to evaluate impact of price, speed and storage of grid resource. The results show that MADA algorithm is better from both resource's and user's perspective providing high resource utilization. The results also highlight how a resource user can improve his performance using multi-attribute characteristics.
\end{abstract}

Keywords: Double auction, grid resource, multi-attribute, satisfaction degree.

\section{INTRODUCTION}

Grid resource computing system is defined as the next generation computing platform to solve large scale problems in science, industry and engineering. Computational grids support the creation of virtual organizations which enable the sharing, exchange, selection, and aggregation of geographically distributed heterogeneous resources [1]. In recent years, business models or economic mechanisms have been proved to be suitable for solving the problem of grid resource allocation [2]. Research on computational grid management can be divided into two categories: commodity market models and auction based models. It has been proved that the auction models are more suitable than commodity market models for the gird resource allocation for its advantages of needing little information, with decentralized structure and more easily to implement [3].

Research on resource management for grid computing can be divided into two categories: commodity market models and auction based models. In the commodity market models, the transaction price is a constant price determined by the total quantity of supply and demand. While in auction based models, the buyers and sellers are independent from each other who agree privately on the selling price. It has been proved that the auction models are more suitable than commodity market models for the gird resource allocation for its advantages of needing little information, with decentralized structure and more easily to implement. Among the traditional auction models and mechanisms, the double auction has a high potential for grid computing [4]. In a double auction model, consumers submit bids and providers submit requests at any time during the trading period. If at any time there are bids and requests that match or are compatible with a price, then a trade is executed immediately. Suri and Singh

*Address correspondence to this author at the 579 Qianwangang Road Economic \& Technical Development Zone, Qingdao Shandong Province, 266590 P.R. China; Tel: 13969872127; E-mail: wlkang@163.com
[5] presented a modified continuous double auction algorithm and gave some results that modified algorithm was significantly better than the traditional CDA in terms of reduction in price volatility. Li et al. [6] studied the combinatorial double auction, and designed a novel pricing algorithm for the allocation model to obtain the detailed resource allocation status and the price information. Xia et al. [7] presented that the combinatorial double auction not only had the advantages of the combinatorial auction, but also considered the requirements of both buyers and sellers. Fang et al. [8] established a double auction based Bayesian game with power supplier and demander in open bilateral electricity market, solved the Bayesian Nash equilibrium and gave the equilibrium bidding strategies for power supplier and demander. Prodan et al. [9] designed a novel double auction mechanism which was appropriate for allocation of multiunit homogenous and divisible resources in the Grid. Kant and Ding et al. [10] proposed the double auction allocation model for grids, and three double auction algorithms for resource allocation were provided. Garg et al. [11] presented an improved grid resource management model based on double auction and proposed bidding strategies to resolve the low efficiency problem. The simulation results showed that the price strategy could improve the efficiency of grid resource auction.

Different from the original literature, in this paper, the multi-attribute characteristics of computational grid are introduced into the double auction model. In reality, the computer system, network subsystem, file system and database system compose many different resources types. Each resource type has one or more attributes with specific values, such as price, computational speed and storages. Therefore, the attributes of price, speed and storage may influence the auctioneer's decision, simultaneously. In our approach, we design Single Price Double Auction algorithm (SPDA), and Multiple Attribute Double Auction algorithm (MADA). The fairness and comprehensive performance are introduced into models to help achieve resource utilization maximization 


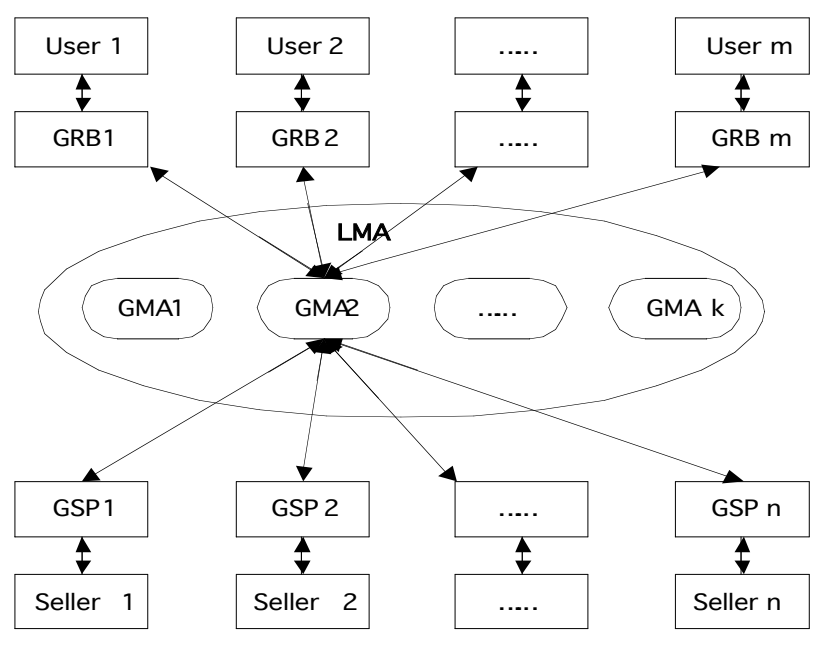

Fig. (1). Overview of the architecture.

based on multi-attribute bids. The simulating experiments show that MADA algorithm has good behavior in grid environment. It has better performance on user satisfaction level and market information efficiency.

The paper is organized as follows. Section 2 presents the formulation about multi-attribute double auction. In section 3 , the SPDA and MADA algorithms are provided based on the multi-attribute bids. The simulating experiment results are presented in section 4 . In section 5 we draw conclusions and present future research directions.

\section{PROBLEM FORMULATION}

There are three participants in our grid environment. In the following part, we present each of these participants and describe their roles in the algorithms and their characteristics in Fig. (1).

Grid Resource Broker (GRB): GRB acts on behalf of the resource user, i.e., the buyer. The job of GRB is to announce the basic attributes, the required quantities and the asking price of the grid resource. In our model, we assume that each grid resource buyer $i$ has one independent computational intensive job $J_{i}^{(b)}$ for execution and is willing to pay for it. The $J_{i}^{(b)}$ is denoted by a fourtuple $J_{i}^{(b)}=\left(L_{i}^{(b)}, \mathrm{T}_{i}^{(b)}, R P_{i}^{(b)}, S_{i}^{(b)}\right)$, where $L_{i}^{(b)}$ is the length of the job of the $i^{\text {th }}$ resource buyer and is specified by millions of instructions (MI), $\mathrm{T}_{i}^{(b)}$ determines the job deadline, $R P_{i}^{(b)}$ represents the budget allocated to $J_{i}^{(b)}$ and $S_{i}^{(b)}$ is the minimum storage. Each buyer aims at executing its job within its corresponding deadlines and minimizing the cost. During the auction period, each GRB $i$ also present request denoted by a two-tuple $\left(a_{i}^{(b)}, q_{i}^{(b)}\right)$, where $a_{i}^{(b)}$ is the asking price and $q_{i}^{(b)}$ is the required quantity.

Grid Service Provider (GSP): GSP acts on behalf of the resource provider, i.e., the seller. The job of GSP is to compute the availability of the current resource, estimate the storage capacity and calculate the bidding price of grid resource. In the double auction mechanisms, firstly GRB notifies the specific attribute requirements of computational grid resource. GSP arrives dynamically with his supply ability, accept task and submit his bid. Suppose that GSP $j$ 's multi-attribute bid $B_{j}^{(s)}$ is denoted by $B_{j}^{(s)}=\left(b i d_{j}^{(s)}, q_{j}^{(s)}, v_{j}^{(s)}, s_{j}^{(s)}\right)$, where $b_{i d}{ }_{j}^{(s)}$ is the bidding price of the $j^{\text {th }}$ GSP, $q_{j}^{(s)}$ denotes the supply quantity of grid resource by the $j^{\text {th }} \mathrm{GSP}, v_{j}^{(s)}$ is the computational speed of resource and is expressed in terms of millions of instructions in one second (MIPS) and $s^{(s)}$ denotes the attribute of storage capacity of the grid resource.

Grid Market Auctioneer (GMA): GMA is the auctioneer of the double auction. The job of GMA is to use the double auction algorithm to control the behavior of auction market and decide the final winners of the auction.

In this paper a comprehensive index of QoS, i.e., the satisfaction degree is introduced into the double auction model. For the given GSP $j$ 's multi-attribute, the GMA calculates the utility denoted by $U_{j}^{(s)}$ according to the following satisfaction degree;

$$
U_{j}^{(s)}=w_{1} \frac{R P_{i}^{(b)}-b i d_{j}^{(s)}}{R P_{i}^{(b)}}+w_{2} \frac{T_{i}^{(b)}-\frac{L_{i}^{(b)}}{v_{j}^{(s)}}}{T_{i}^{(b)}}+w_{3} \frac{s_{j}^{(s)}-S_{i}^{(b)}}{s_{j}^{(s)}}
$$

where $0 \leq w_{1} \leq 1,0 \leq w_{2} \leq 1$ and $0 \leq w_{3} \leq 1$ indicate the weights of computational speed, price and memory size, respectively. It is clear that $w_{1}+w_{2}+w_{3}=1$.

Local Market for Auctions (LMA): GRB, GSP and GMA are three intelligent entities having their own specific objectives in LMA. GRB and GSP interact with GMA through Local Market for Auction (LMA). LMA provides support for GRB to post their characteristics, and enables GMA to find the right resources that the task needed. LMA takes a request for a task from a GRB specified in an appro- 


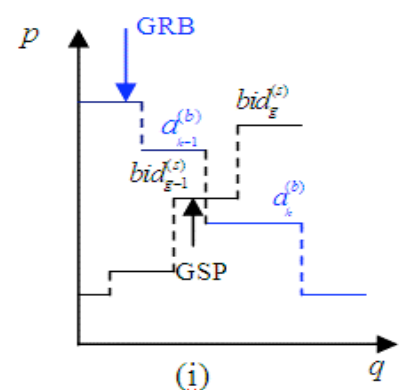

Fig. (2). Illustration of matching.

priate language and returns the list of resources that match the requirements of the task.

The resource users use the GRBs to create a valid schedule (1). Resource providers use GSPs in order to connect their local scheduling systems to the GMAs (2). The GRBs and GSPs interact to maximize the utilities of their owners in the Local Market for Auctions (LMA) (3).

\section{MULTI-ATTIRIBUTE DOUBLE AUCTION MOD- ELS AND ALGORITHMS}

We assume that there are $m$ GRBs (buyers) and $n$ GSPs (sellers) to participate in double auction. After GRBs and GSPs send the information of their prices to GMA within the limited time, GMA collects and sorts all bidding and asking prices. Generally, the bidding price and asking price sequences satisfy that $b i d_{1}^{(s)} \leq b i d_{2}^{(s)} \leq \ldots \ldots \leq b i d_{\mathrm{n}}^{(s)}$ and $a_{1}^{(b)} \geq a_{2}^{(b)} \geq \ldots \ldots \geq a_{\mathrm{m}}^{(b)}$, respectively, i.e., the demand prices are decreasing and the supply prices are increasing. We describe these characters in Fig. (2), in which the horizontal axis denotes the quantity $q$ and the vertical axis denotes the price $p$ of grid resource. The point where these two curves intersect is the equilibrium price, i.e., the $(g-1)^{\text {th }}$ GSP's bidding price bid $_{g-1}{ }^{(s)}$ is the final trading price or temporary winning price (see details in the following subsections).

In Fig. (2), there are three cases about the equilibrium price, i.e., (i) $a_{k}^{(b)} \leq b i d_{g-1}^{(s)} \leq a_{k-1}^{(b)}$, (ii) $b i d_{g-1}^{(s)} \leq a_{k-1}^{(b)} \leq b i d_{g}^{(s)}$, (iii) $b i d_{g-1}^{(s)} \leq a_{k-1}^{(b)}$ and $a_{k}^{(b)} \leq b i d_{g}^{(s)}$. Considering fairness, the GSPs before $(g-1)$ all become the final or temporary winners and the GRBs before $(k-1)$ all become the final winners in the double auction. We assume the trading price of the double auction is $p$ and the final transaction quantities of each GRB and GSP are $Q_{i}^{(b)}$ and $Q_{j}^{(s)}$, respectively.

In the following subsections, we describe the Single Price Double Auction algorithm (SPDA), in which the winners are decided only depending on the price-attribute and the Multiple Attribute Double Auction algorithm (MADA), in which multiple attributes are deciding factors.

\subsection{SPDA Algorithm}

Step 1: GRBs and GSPs send the information of their prices and quantities to GMA within the limited time.
Step 2: GMA accepts and sorts the bidding prices and the asking prices. According to rules about the equilibrium price in Fig. (2), GMA determines the final winners of GRB and GSP in the double auction.

Step 3: The final trading price is calculated by the following equation:

$$
p=\frac{b i d_{g-1}^{(s)}+a_{k-1}^{(b)}}{2} \text {. }
$$
rules.

The trading quantities are allocated by the following

Case 1: If the demand and supply quantities of GRBs and GSPs satisfy $\sum_{j=1}^{g-1} q_{j}^{(s)} \leq \sum_{i=1}^{k-1} q_{i}^{(b)}$, it means that demand exceeds supply. Therefore, the trading quantities of $j^{\text {th }}$ winning GSP is $Q_{j}^{(s)}=q_{j}^{(s)}$ and the trading quantities of $i^{\text {th }}$ winning GRB is:

$Q_{i}^{(b)}=q_{i}^{(b)}\left(\sum_{j=1}^{g-1} q_{j}^{(s)} / \sum_{i=1}^{k-1} q_{i}^{(b)}\right)$.

Case 2: If the demand and supply quantities of GRBs and GSPs satisfy $\sum_{j=1}^{g-1} q_{j}^{(s)}>\sum_{i=1}^{k-1} q_{i}^{(b)}$, the supply exceeds demand. Therefore, the trading quantities of $j^{\text {th }}$ winning GSP is $Q_{j}^{(s)}=q_{j}^{(s)}\left(\sum_{i=1}^{k-1} q_{i}^{(b)} / \sum_{j=1}^{g-1} q_{j}^{(s)}\right)$. The trading quantities of $i^{t h}$ winning GRB is $Q_{i}^{(b)}=q_{i}^{(b)}$.

Step 4: GMA informs each GRB and GSP the results of the double auction, e.g., winner or loser, trading price and quantities.

Step 5: The winning GSPs provide grid resources to the winning GRBs, and GRBs pay for its service.

\subsection{MADA Algorithm}

Different from the SPDA algorithm, we present novel rules about the final winners based on the request of satisfaction degree about QoS of computational grid resource providers. Let $\Gamma$ and $F$ denote the sets of temporary and final winning GSPs, respectively. Suppose that $\mathrm{K}$ is the set of the final winning GRBs. The MADA algorithm is as follows: 
Step 1: GRBs and GSPs send the information of their prices, quantities and other required attributes, e.g., speed, time and memory size to GMA within the limited time.

Step 2: GMA accepts and sorts the bidding prices and the asking prices. The final winning GRSs and the temporary winners of GSPs are made sure based on the equilibrium price rules in Fig. (2).

Step 3: GMA determines the final winners among the temporary winners of GSPs according to the comprehensive utility computed by satisfaction degree.

The final trading price is calculated by comparing the $(g-1)^{\text {th }}$ GSP's utility with the $g^{\text {th }}$ GSP's utility, that is:

$p=\left\{\right.$ bid $_{j}^{(s)} \mid \max \left(U_{g-1}^{(s)}\left(\right.\right.$ bid $\left._{j}^{(s)}\right), U_{g}^{(s)}\left(\right.$ bid $\left.\left.\left._{j}^{(s)}\right)\right)\right\}$.

Furthermore, if the utility of each temporary winning GSP satisfies $U_{j}^{(s)} \geq \max \left\{U_{g-1}^{(s)}, U_{g}^{(s)}\right\}$, the temporary winning GSPs become the final winner, else they become the losers.

Step 4: GMA allocates the trading quantities for both winning GRBs and GSPs by the following rules.

Case 1: When the demand exceeds supply, i.e., $\sum_{j \in F} q_{j}^{(s)}-\sum_{j \in \Gamma} q_{j}^{(s)} \leq \sum_{i \in \mathrm{K}} q_{i}^{(b)}$, the trading quantities of $j^{\text {th }}$ winning GSP is $Q_{j}^{(s)}=q_{j}^{(s)}$ and the trading quantities of $i^{\text {th }}$ winning GRB is:

$Q_{i}^{(b)}=q_{i}^{(b)}\left\{\left(\sum_{j \in F} q_{j}^{(s)}-\sum_{j \in \Gamma} q_{j}^{(s)}\right) / \sum_{i \in \mathrm{K}} q_{i}^{(b)}\right\}$.

Case 2: When the supply exceeds demand, i.e., $\sum_{j \in F} q_{j}^{(s)}-\sum_{j \in \Gamma} q_{j}^{(s)}>\sum_{i \in \mathrm{K}} q_{i}^{(b)}$, the trading quantities of $j^{\text {th }}$ win- ning GSP is $Q_{j}^{(s)}=q_{j}^{(s)}\left\{\sum_{i \in \mathrm{K}} q_{i}^{(b)} /\left(\sum_{j \in F} q_{j}^{(s)}-\sum_{j \in \Gamma} q_{j}^{(s)}\right)\right\}$ and the trading quantities of $i^{\text {th }}$ winning GRB is $Q_{i}^{(b)}=q_{i}^{(b)}$.

Step 5: GMA informs each GRB and GSP the result of the double auction.

Step 6: The winning GSPs provide grid resources to GRBs, and GRBs pay for their services.

\section{SIMULATION AND EXPERIMENTAL RESULTS}

In this section the simulations are given to describe the SPDA algorithm and MADA algorithm. In our simulation, we assume the grid resource is PC, i.e., there are 20 grid PC buyers and 30 PC sellers, the length of the jobs are considered as a random integer within the range [5000, 15000] MZ sampled from a uniform distribution. Also the computational capacity of GRB's request and GSP is normally distributed within the range $[100,500] \mathrm{MZ} / \mathrm{s}$, storage capacity distributed within $[1,8] \mathrm{G}$. The price that resource provider can be accepted over $[50,250]$ G\$/MIPS, the price that resource buyer can offer distributed over [100,200] G\$/MIPS. The quantities that GSPs can provide and GRBs required are both within the range of $[10,20]$. The utility of each GSP's asking bid is set according to Eq.1. Also in our experiments, we assume that $w_{1}=0.3, w_{2}=0.4$ and $w_{3}=0.3$.

We simulate the process of the multi-attribute double auction, in which each point has two axes, $x$ label denotes the total quantities that GRBs required or GSPs can provide and $y$ label denotes the prices that GRBs bidding and GSPs asking to the GMA. From Fig. (3), we can know that the bidding price of the $12^{\text {th }} \mathrm{GRB}$ is between the asking price of the $14^{\text {th }}$ and $15^{\text {th }}$ GSP, belongs to the second situation as mentioned in Fig. (2). Then we compare the utility of the $14^{\text {th }}$ and the $15^{\text {th }}$ GSP, i.e., $U_{14}^{(s)}>U_{15}^{(s)}$. The result is that the asking price of the $14^{\text {th }}$ GSP is the unite price of the double auction.

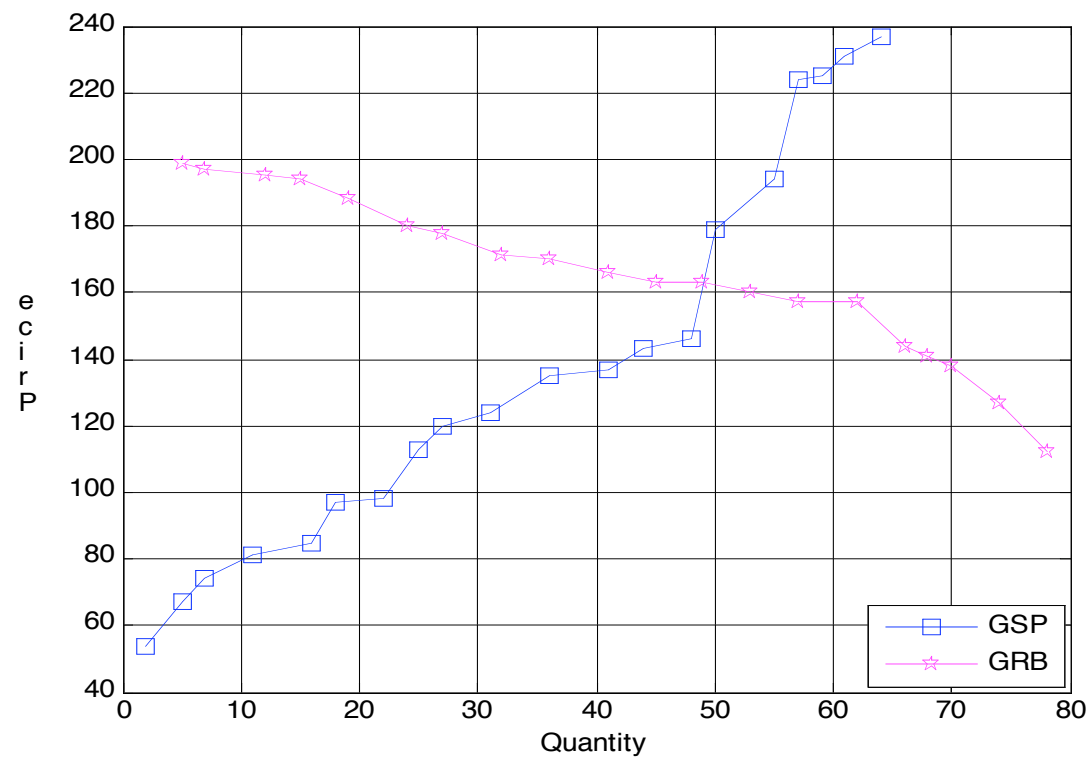

Fig. (3). The process of sort on price. 


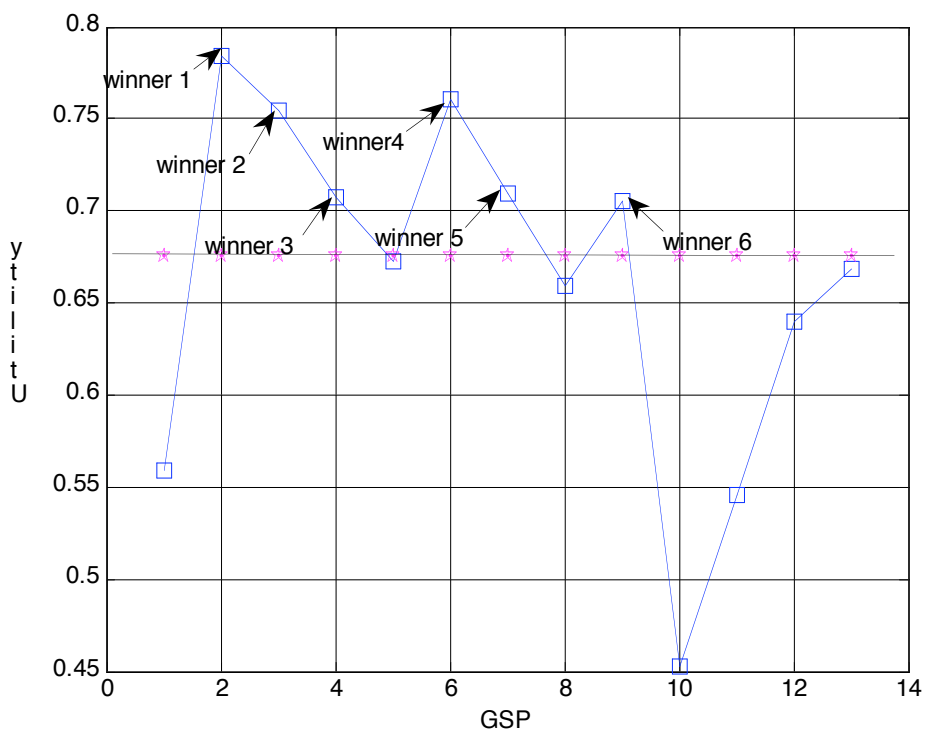

Fig. (4). Diagram of winners.

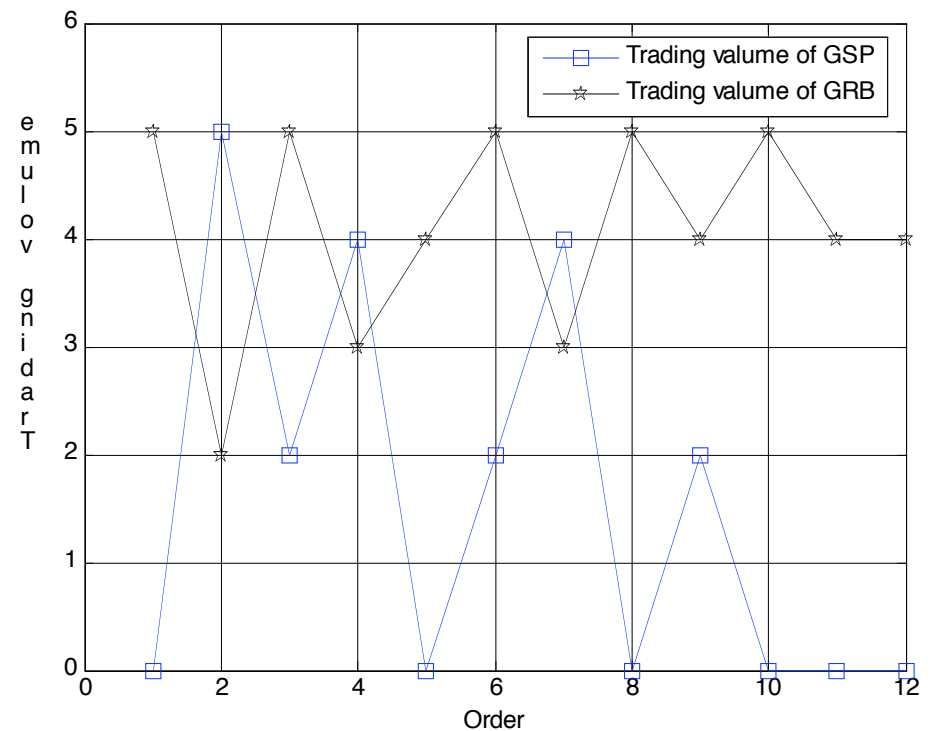

Fig. (5). Trading quantities of GRBs and GSPs.

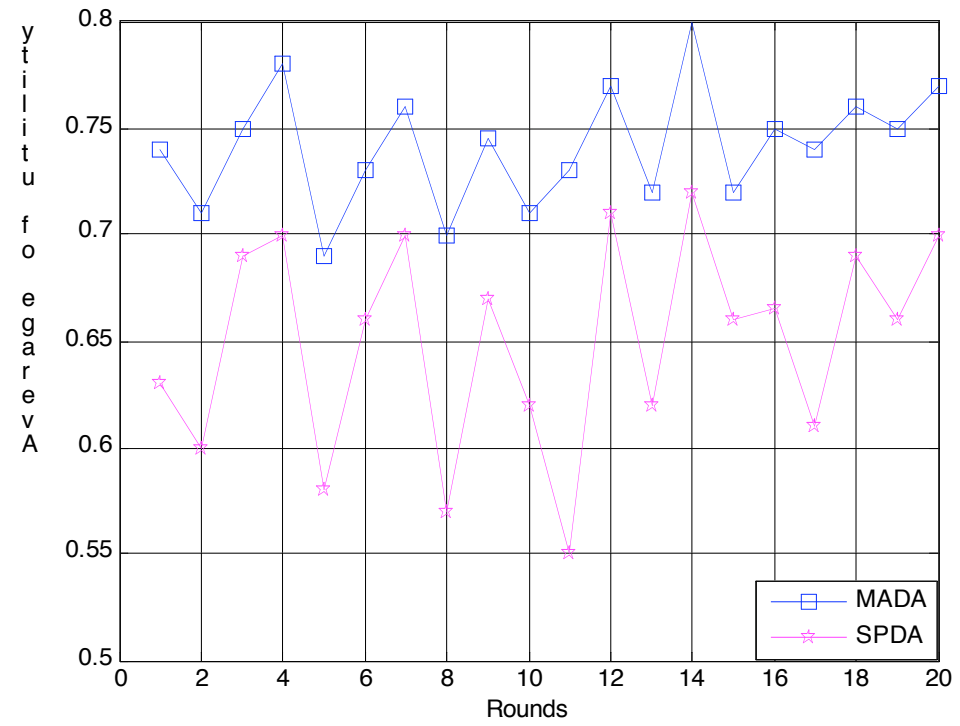

Fig. (6). Comparison of average utilities. 
The GRBs and GSPs before the intersection compose the sets of temporary winners of the double auction. In Fig. (4), we choose the final winners of the multi-attribute double auction based on the utility of $U^{(s)}$. As shown in Fig. (4), the black line denotes the value of $U_{14}^{(s)}$, the GSPs whose utilities are larger than $U_{14}^{(s)}$ become the final winners, i.e., the winners are from 1 to 6 .

According to the equation mentioned before, we simulate the trading quantities of GRBs and GSPs in Fig. (5). For simplicity, we only describe such case that the supply exceeds demand.

In Fig. (6), this paper compares the average utilities of the SPDA algorithm and MADA algorithm. As Fig. (6) shows that the average utilities of MADA algorithm is higher than that of SPDA algorithm. This highlights that fairness and satisfaction degree can help GRBs choose final winners in the double auction models and achieve more effective in improving the buyer's utility.

\section{CONCLUSION}

In this paper, we propose an improved double auction model. Firstly, we investigate multi-attribute characteristics of grid resource in the double auction models and present two algorithms to describe the matching processes based on double auction. The simulations show that the method this paper proposed is much more efficient than the previous methods in satisfying the utility of the grid resource buyers. The experimental results clearly illustrate that the proposed method is efficient and can be used for solving such problems.

\section{CONFLICT OF INTEREST}

The authors confirm that this article content has no conflict of interest.

\section{ACKNOWLEDGEMENTS}

This work is supported by the NSFC (71371111; 71471105), Humanity and Social Science Youth foundation of Ministry of Education of China (14YJA630007), Social Science Fund of Shandong (13CGLZ03).

\section{REFERENCES}

[1] A. Attanasioa, G. Ghianib, L. Grandinettia, and F. Guerrieroa "Auction algorithms for decentralized parallel machine scheduling," Parallel Computing, vol. 32, pp. 701-709, 2006.

[2] L.L. Ding, X.M. Liu, and Y.F. Xu, "Competitive risk management for online Bahncard problem," Journal of Industrial and Management Optimization, vol. 6, no. 1, pp. 1-14, 2010.

[3] A. Haque, S.M. Alhashmi, and R. Parthiban, "Towards better understanding of two economic models: A grid perspective," Communications of the IBIMA, vol. 2011, pp. 1-11, 2011.

[4] R. Buyya, D. Abramson, J. Giddy, and H. Stockinger, "Economic models for resource management and scheduling in Grid computing," The Journal of Concurrency and Computation, vol. 14, no. 13-15, pp. 1507-1542, 2002.

[5] P. K. Suri, and M. Singh, "Auction oriented resource allocation in grid using knowledge based pricing policy," IJCSNS International Journal of Computer Science and Network Security, vol. 9, no. 9, pp. 91-95, 2009.

[6] L. Li, Y. Liu, K. Liu, X. Ma, and M. Yang, "Pricing in combinatorial double auction-based grid allocation model," The Journal of China Universities of Posts and Telecommunications, vol. 16, no. 3, pp. 59-65, 2009.

[7] M. Xia, J. Stallaert, and B.A. Whinston, "Solving the combinatorial double auction problem," European Journal of Operational Research, vol. 164, no. 1, pp. 239-251, 2005.

[8] D. Fang, X. Wang, Y. Zhang, K. Liu, and P. Wang, "Double auction bayesian model with supplier and demander in open bilateral electricity market," Power System Technology, vol. 27, no. 12, pp. 1-5, 2003.

[9] R. Prodan, M. Wieczorek, and H.M. Fard, "Double auction-based scheduling of scientific applications in distributed grid and cloud environments," Journal of Grid Computing, vol. 9, no. 4, pp. 531548, 2011.

[10] L.L. Ding, W.L. Kang, and X.M. Liu, "An Interactive Approach for multi-attribute resource allocation in grids," Journal of Software vol. 8, no. 10, pp. 2607-2611, 2013.

[11] S.K. Garg, S. Venugopal, J. Broberg, and R. Buyya, "Double auction-inspired meta-scheduling of parallel applications on global grids," Journal of Parallel and Distributed Computing, vol. 73, no. 4, pp. 450-464, 2013.

\author{
Received: October 16, 2014 \\ Revised: December 23, 2014 \\ Accepted: December 31, 2014 \\ (C) Kang et al:; Licensee Bentham Open.
}

This is an open access article licensed under the terms of the Creative Commons Attribution Non-Commercial License (http://creativecommons.org/licenses/by-nc/3.0/) which permits unrestricted, non-commercial use, distribution and reproduction in any medium, provided the work is properly cited. 